\title{
La práctica profesional docente, un dispositivo contextualizado para la formación en las licenciaturas de la Fundación Universitaria San Alfonso*
}

\author{
Leina Lucelva García Reina \\ Fundación Universitaria San Alfonso, Bogotá, Colombia \\ leina.garcia@usanalfonso.edu.co \\ https://orcid.org/0000-0001-5101-2990
}

\section{RESUMEN}

Las diferentes concepciones acerca de la práctica docente van determinando su relación directa con los paradigmas prevalecientes en el campo educativo, en donde cada uno da cuenta de un sustrato epistemológico específico. Del análisis documental elaborado, como técnica de investigación cualitativa, se puede inferir que la práctica docente ha pasado de una relación transmisión-recepción, propia de los enfoques tradicionales, a una relación enmarcada dentro de una racionalidad crítica o emancipatoria, como lo es el enfoque reflexivo-investigativo. Esta última guía sistemática - que orienta el proceso de enseñanza - permite concebir la práctica docente como un espacio que logra, por una parte, el reconocimiento de la cultura escolar emergente a través de la diversidad de rituales incorporados en la cotidianidad escolar, y que se convierten en dispositivos que posibilitan leer la vida de la escuela en perspectiva compleja, distinguiendo entre lo estructural y lo contingente; y por otra, reconocer la praxis docente como una práctica investigativa que ofrece la posibilidad de generar nuevo conocimiento Esta nueva manera de concebir el ejercicio docente desde la teoría pedagógica se reconoce como experiencias contextualizadas o situadas, conducentes a formar un profesional que se reconozca como mediador, reflexivo, crítico e intelectual, para enfrentar los retos pedagógicos y sociales que le demanda el medio.

Palabras clave: práctica docente; formación inicial docente; saber profesional; práctica contextualizada.

Cómo citar: García Reina, L. (2021). La práctica profesional docente, un dispositivo contextualizado para la formación en las licenciaturas de la Fundación Universitaria San Alfonso. Ciencias Sociales y Educación, 10(20), 169-195. https://doi.org/10.22395/csye.v10n20a8 Recibido: 15 de diciembre de 2020.

Aprobado: 24 de marzo de 2021. 


\section{Teaching Professional Practice, a Contextualized Device for Training in Bachelor's Degrees at the San Alfonso University Foundation}

\section{ABSTRACT}

The different conceptions about teaching practice determine its direct relationship with the prevailing paradigms in the educational field, each one with its specific epistemological substratum. From the elaborate documentary analysis, as a qualitative research technique, it can be inferred that teaching practice has gone from a transmission reception relationship, typical of traditional approaches, to a relationship framed within a critical or emancipatory rationality, such as the reflective investigative approach. This approach makes it possible to conceive teaching practice as a space that achieves, on the one hand, the recognition of the emerging school culture through the diversity of rituals incorporated in the daily school life, and which become devices that make it possible to read the life of the school from a complex perspective, distinguishing between the structural and the contingent, and on the other, recognizing teaching practice as an investigative practice that offers the possibility of generating new knowledge. This new way of conceiving practices from pedagogical theory is recognized as contextualized or situated practices, leading to forming a professional who is recognized as a mediator, reflective, critical and intellectual, to face the pedagogical and social challenges that the environment demands.

Keywords: teaching practice; initial teacher training; professional knowledge; contextualized practice. 


\section{Prática profissional de ensino, um dispositivo contextualizado para a formação de bacharelados na Fundação Universidade San Alfonso}

\section{RESUMO}

As diferentes concepções sobre a prática docente determinam sua relação direta com os paradigmas vigentes no campo educacional, cada um com seu substrato epistemológico específico. A partir da elaborada análise documental,como uma técnica de pesquisa qualitativa, podese inferir que a prática docente passou de uma relação transmissão-recepção, típica das abordagens tradicionais, para uma relação enquadrada em uma racionalidade crítica ou emancipatória, como a abordagem reflexivo-investigativa. Essa abordagem possibilita conceber a prática docente como um espaço que consegue, por um lado, o reconhecimento da cultura escolar emergente por meio da diversidade de rituais incorporados ao cotidiano escolar, e que se tornam dispositivos que possibilitam a leitura da vida dos a escola numa perspectiva complexa, distinguindo o estrutural e o contingente $e$, por outro lado, reconhecendo a prática docente como uma prática investigativa que oferece a possibilidade de gerar novos conhecimentos. Essa nova forma de conceber as práticas a partir da teoria pedagógica é reconhecida como práticas contextualizadas ou situadas, levando a formar um profissional reconhecido como mediador, reflexivo, crítico e intelectual, para o enfrentamento dos desafios pedagógicos e sociais que o meio ambiente exige.

Palavras chave: prática docente; formação inicial de professores; conhecimento profissional; prática contextualizada. 


\section{Introducción}

A partir del ejercicio investigativo realizado hasta el momento presentamos un avance que atiende a la pregunta sobre cómo se ha concebido la práctica docente en diferentes épocas de su desarrollo teórico en el escenario nacional. Proponemos aterrizar esta pregunta en el escenario institucional, acudiendo a un proceso sistemático y procesual de recolección de información. La metodología que utilizamos implica la selección, la clasificación, el análisis y la evaluación de contenidos presentes en los diferentes documentos abordados; esto nos permite ofrecer, como resultado del proceso investigativo, las bases que sostienen el diseño final del modelo pedagógico para la práctica docente.

Para poder llegar al diseño del modelo pedagógico para la práctica docente se necesita partir del reconocimiento de la práctica docente como un espacio idóneo tanto de enseñanza como de aprendizaje. Por ende, se hace necesario convocar a los programas de licenciatura a reflexionar desde la escuela y para ella. Esto supone analizar las dinámicas del proceso docente educativo presentes en el aula y en la institución según: los contextos, la relación dialógica entre lo sociohumanístico y lo disciplinar, en donde esta última incluya lo pedagógico y lo investigativo; los referentes políticos, culturales y sociohistóricos que la disciplina encierra; los contenidos curriculares y las competencias en las que se está formando como futuro profesional de la educación, y con acentuado énfasis en la actualidad, a reflexionar acerca de las propias prácticas docentes como eje transversal investigativo.

En esta dirección, el ejercicio en cuestión puede mostrar variaciones de acuerdo con la tendencia epistemológica de los programas, aunque de manera general, lo importante es que esto conlleve a tomar conciencia que las prácticas docentes deben apreciarse como espacio de reflexión.

Acompañamos esta reflexión con algunas concepciones sobre la práctica profesional de los docentes en formación inicial encontradas en los diferentes contextos analizados a partir de los documentos seleccionados a nivel nacional e institucional. Con esto, se abrió la posibilidad de ubicar aportes significativos para la comprensión de algunas situaciones problemáticas, que para el caso, se definen a partir de dos perspectivas: i) desde las exigencias de la normatividad y ii) desde lo que la institución diseña en el programa académico de la licenciatura.

Al tener en cuenta el análisis documental, consideramos algunos criterios en relación con: i) una mirada teórica y metodológica muy reducida, realizada por parte de las autoridades educativas acerca de la concepción de lo que es un modelo de práctica docente — que lo establecen como de carácter obligatorio-y su correspondencia con las demandas actuales de la ciencia pedagógica, 
ii) poco abordaje de la práctica docente como acción social y con carácter históricamente determinado y iii) la no existencia de un modelo pedagógico, como problemática frecuente en las IES, científicamente argumentado, que dé cuenta de las cualidades de la práctica docente para su desarrollo.

Las anteriores situaciones problemáticas permiten a la investigadora identificar una contradicción fundamental que se establece entre los requerimientos que la normatividad exige y lo que la institución propone curricularmente para el desarrollo de una práctica docente planificada, organizada e históricamente situada que necesita enmarcarse dentro de un proyecto en perspectiva humanista, reflexiva, crítica y desarrolladora, y que conlleve a experimentar una práctica ajustada a la realidad social y al componente normativo.

A partir de esta contradicción se formula el siguiente problema científico que, aunque se presenta atendiendo al caso particular de una IES de Colombia, la investigación puede servir para hacer un análisis en diferentes IES del país. Así que el problema se define de la siguiente manera: ¿cómo contribuir al desarrollo de la práctica docente en la Licenciatura en Filosofía en la Fundación Universitaria San Alfonso de Bogotá, Colombia en correspondencia con las exigencias socioculturales colombianas? En esta vía, la investigación asume un doble objeto de estudio: por un lado, la práctica docente en la formación inicial; y, por el otro, propone un modelo pedagógico para el desarrollo de la práctica docente en la licenciatura de Filosofía en la Fundación Universitaria San Alfonso de Bogotá, Colombia en correspondencia con las exigencias socioculturales colombianas. Es necesario señalar que el modelo pedagógico, como resultado de la investigación, puede entrar en diálogo con otras IES a nivel local o regional.

Atendiendo a los anteriores presupuestos, iniciaremos por reconocer las concepciones teóricas frente a la práctica. Como se anotó en algún apartado anterior, estas cobra gran importancia. De igual manera determinaremos cómo ha evolucionado la concepción de la práctica docente en la formación inicial dentro del sistema educativo, hasta lograr ubicar dicha concepción en el contexto institucional. Esto permite fundamentar cada uno de los sistemas que van a ser parte estructural del modelo.

Por otra parte, es importante para este estudio reconocer que, en el contexto colombiano, los diferentes análisis sobre las prácticas profesionales de docentes de formación o aquellas reconocidas en muchos programas como prácticas pedagógicas, son un tema de nuevo de interés entre los Investigadores. Más aún, cuando las reformas educativas tendientes a establecer las características específicas de calidad para los programas de licenciatura, establecen exigencias muy específicas en donde los valores, los conocimientos y las competencias del educador se enmarcan dentro de componentes que articulados entre sí, le ofrecen 
al docente en formación, las herramientas suficientes y necesarias para desenvolverse en ese espacio de acción y reflexión como es la práctica.

Se hizo una aproximación a diversos autores del ámbito nacional como María Angulo y José Álvarez (2010), Pedro Baquero y Wilmer Villa (2013), Martha Helena Baracaldo (2007). Para este análisis se llevó a cabo atendiendo a situaciones previas que enmarcan el estudio como la selección del tema, la delimitación de este, el planteamiento del problema, la búsqueda de teorías que expliquen el fenómeno, la selección de fuentes de información, la operacionalización del proceso y la focalización de hallazgos frente a las concepciones sobre las prácticas docentes en la formación inicial del educador.

Es importante destacar cómo en los estudios analizados, cuyo transcurrir va desde la década de los noventa hasta la actualidad, la práctica docente es concebida desde tres miradas convergentes: el profesor reflexivo, el profesor investigador y el profesor mediador. Así, para dar cuenta del propósito central, este artículo se precisa la siguiente información: i) la historia de la práctica docente en la formación inicial en Colombia; ii) la concepción de la práctica docente para la Fundación Universitaria San Alfonso; su definición operacional y todo aquello que hace parte del horizonte institucional, el cual orienta el desarrollo de cada una de las funciones sustantivas de la misma. iii). Y, finalmente, las conclusiones.

\section{Historia de la práctica docente en la formación inicial en Colombia}

De la misma manera como se han identificado los múltiples factores que han definido y orientado el desarrollo epistemológico de la práctica docente en un ámbito global, se reconoce de igual manera un contexto y un devenir histórico para la misma en Colombia, entorno inmerso en las realidades de la sociedad occidental de la segunda mitad del siglo XX. Estas nuevas corrientes de pensamiento permearon los procesos educativos que promovieron la promulgación de la Ley General de Educación o Ley 115 (1994) que en el articulado que va desde el 109 hasta el 114 habla específicamente de la formación de los educadores, en donde ya el énfasis no está dado por el ejercicio de la didáctica, los contenidos, las tecnologías y el currículo, sino, por el contrario, centra su interés en los fines de la formación de los educadores, en el mejoramiento profesional, en la profesionalización y en la reestructuración de instituciones formadoras de docentes Todo focalizado alrededor de un concepto central como es la autonomía.

De ahí, la importancia de reconocer el proceso de desarrollo del sistema educativo a partir de la década de los años sesenta en el contexto colombiano y, específicamente, en lo que refiere a la práctica docente en la formación inicial, 
que es el interés de la presente investigación, junto con el propósito de definir las tendencias desde las cuales se ha desarrollo dicho proceso.

En este orden de ideas, el estudio adelantado por el Ministerio de Educación Nacional de Colombia (2007) titulado: investigación de los saberes pedagógicos, bajo la coordinación de Martha Helena Baracaldo Quintero, ofrece un recorrido muy interesante alrededor de los hitos que marcaron la historia de la formación docente, específicamente, en cómo se concebía el rol del educador y, por supuesto, alrededor de qué concepto central eran puestos en escena los saberes adquiridos durante su formación.

A nivel del sistema educativo colombiano desde la década de los años sesenta, se vienen perfilando acciones para dar respuesta a las diferentes problemáticas de carácter complejo que se presentan en el contexto escolar. En los últimos años de esta década, producto del diagnóstico elaborado por la misión pedagógica alemana, se diseñaron estrategias que tenían como foco de actuación la planificación. Estas decisiones implicaron:

la introducción de la planificación al sector educativo desplazando las antiguas prácticas pedagógicas de los maestros; la práctica del maestro se esquematiza haciendo de la enseñanza un modelo técnico que puede ser guiado por pasos precisos. La enseñanza se fragmenta, se parcela, se dosifica en paquetes distribuidos por temas, objetivos, actividades, recursos y evaluación en meses y semanas; la introducción de nociones como objetividad, confiabilidad, efectividad y eficacia en el discurso y prácticas pedagógicas. (Anacona et al., 2007, pp. 63-64)

Es decir, la tendencia es la de reconocer la práctica docente como una actividad preelaborada, preconcebida, preformulada, con adiestramientos sistemáticos y con controles periódicos. La participación del docente desde sus saberes es mínima en la configuración del proceso enseñanza aprendizaje. La actividad práctica pretende ser eficaz y rigurosa, por tanto, esta es específicamente una aplicación de la teoría. El ejercicio docente está arraigado en las estructuras administrativas, en los hábitos y en la distribución de roles. Concepción de docente técnicoartesanal, en el oficio de ser maestro.

A finales de los setenta, inicia un proceso de instrumentalización

representado en las nociones de "práctica pedagógica" que se manifiestan en [un] Manual de Práctica Docente y que hacían referencia a las posturas e imágenes del maestro, a las ayudas educativas, la planeación y objetivación de la educación, hechos que llevaron a que el saber pedagógico se fundamentara en la operativización en el aula, a partir de su utilización como recurso esencial en la labor del maestro. (Anacona et al., 2007, p. 65) 
Se puede reconocer como tendencia de la práctica docente la denominada por Feiman-Namser (como se citó en Pavié, 2012), de orientación tecnológica, en donde plantea que:

aprender a enseñar supone la adquisición de principios y prácticas derivadas del estudio científico de la enseñanza. El profesor es concebido como un técnico y se aboga un modelo de entrenamiento totalmente estructurado que puede aplicarse a la adquisición de distintas destrezas y conductas que se necesitan. (p. 53)

Se puede reafirmar entonces la anterior condición de la práctica docente desde las palabras de Habermas (citado por Pavié, 2012): "la actividad profesional está dirigida a la solución de problemas mediante la rigurosa aplicación de teorías y técnicas científicas, convirtiéndose en una actividad instrumental" (p. 53).

Desde esta perspectiva, el docente encontrará siempre una respuesta metodológica en un manual que le ayudará a resolver una situación presente en el aula de clase y, por tanto, acudirá al instructivo para obtener la conducta (resultado) deseada en los estudiantes. Se reconoce entonces que: "[e]l andamiaje práctico que soportaba el Manual constituía, entonces, el dispositivo legal (institucionalizado) para asegurar la mecanización del proceso" (Baquero, 2007, p. 11). Se reconoce entonces que el docente desarrolla su profesión como experto técnico.

Continuando con el análisis evolutivo del fenómeno en estudio, a mediados de los años ochenta, la renovación curricular produjo

manuales por grados, en los cuales se encontraba el diseño de asignaturas [microcurrículos] organizadas por objetivos, actividades, metodologías y formas de evaluación, haciendo que el maestro tuviera prácticas alrededor de la administración y reproducción de un currículo externo al contexto escolar, teniendo entonces materializaciones como el currículo diseñado desde el Ministerio de [Educación], el currículo planeado desde el maestro en su planeador, el currículo enseñado representado en lo ejecutado por el maestro en el aula, el currículo aprendido evidenciado en las evaluaciones y el currículo apropiado finalmente por el estudiante, y aplicado en su vida. (Anacona et al., 2007, p. 66)

De esta manera se puede definir que las prácticas están centralizadas en el currículo y en el aprendizaje, y no en la enseñanza. El docente es reconocido desde su actuar como el de "buen empleado", reforzando su rol pasivo y contribuyendo de esta manera en sostener un sistema educativo jerárquico y cerrado. Se afirma a partir de esta perspectiva que la condición del docente, no como un profesional de la educación, sino un operario al servicio de un sistema educativo, es un simple administrador del currículo, despojándolo de su condición de intelectual.

Es importante señalar que en esta década se logra afianzar la idea de microenseñanza como principal dispositivo didáctico de entrenamiento que, a 
su vez, permite avanzar hacia concepciones que buscan articular la docencia, la investigación y el servicio comunitario con la estructura y desarrollo de la práctica docente, denominándose práctica integral. El profesor desarrolla su práctica desde una concepción social que se corresponde con los proyectos sociales de la comunidad.

Las concepciones hasta el momento establecidas pueden ser reconocidas como de corte muy racional/técnico, con algunos vestigios de proyección en lo social. Estas concepciones muestran un salto cualitativo con lo que se propone a partir de la Ley General de Educación (1994). Reza en la Ley que: "una práctica relacionada con el uso de la autonomía y sus posibilidades en el contexto del magisterio oficial; se reconoce la construcción de espacios para la autoformación, la autoevaluación y la vinculación a redes y grupos de maestros investigadores" (Anacona et al., 2007, p. 74).

Todas estas posibilidades permiten que tanto en las normales como en las facultades de educación se construya otro discurso y otras prácticas acerca de la formación inicial docente. Esto proporciona al futuro educador las herramientas suficientes para la construcción de su identidad profesional como educador.

En atención a estas orientaciones, vemos que se pasa de una concepción técnico-artesanal del docente a una concepción práctica de formación profesional. En relación con la práctica docente se pretende que:

los practicantes no sólo [sic] logren comprensiones más holísticas de los fenómenos del aula y de la escuela; sino que además, emprendan iniciativas de intervención de las aulas, que habrán de desembocar en la revisión crítica de los procesos curriculares y didácticos y en la incorporación progresiva del trabajo por proyectos como dispositivo de desarrollo de la práctica docente. (Baquero, 2007, p. 11)

Posterior a esto, el auge de la teoría crítica en las últimas décadas conlleva a la aparición de una nueva perspectiva en la formación inicial docente reconocida como racionalidad crítica o emancipadora. Esta última, a diferencia de las dos anteriores, puede considerarse agenciada por las instituciones educativas: normales y universidades. Así, "esta concepción es más un producto de las dinámicas de la educación popular que de un proyecto académico institucionalizado" (Baquero, 2007).

En palabras de Baquero (2007):

[l]a concepción crítica o emancipadora de las prácticas, vista desde la lógica de la sistematización de experiencias y desde las dinámicas de la investigación-acción permite que el proceso de la práctica se conciba, entonces, como escenario para la acumulación de experiencias generadas desde y para la práctica. (p. 11) 
Resulta en este momento de interés para el estudio, las apreciaciones de Donald Schön (1982) en torno a reconocer desde una postura epistemológica de la práctica profesional, la necesidad de pasar de unas prácticas orientadas desde la visión positivista o de racionalidad técnica a unas prácticas mediadas por la reflexión desde la acción. Esto implica el reconocimiento de que al interior de estas prácticas se generan ciertos estados; en palabras del mismo autor, de incertidumbre, inestabilidad, conflictos, que dan paso a ciertas tensiones en el contexto que por su carácter complejo e inédito, es preciso reconocerlas y generar, de manera consiente, procesos que reivindiquen el papel del docente como profesional crítico y reflexivo que en estos momentos demanda la sociedad. Dicha reflexión requiere del educador una aproximación real a la relación entre teoría/práctica e investigación/ práctica. Se reafirman así los criterios desde los cuáles hoy la normatividad reconoce como de gran importancia frente a la formación docente inicial.

Esta tesis de Schön (1982) son retomadas por Baquero (2007) y en sus propias palabras expresa como esta perspectiva

pone el énfasis en el desarrollo de los procesos, permitiendo que el practicante evidencie desde la recuperación crítica de sus propias experiencias, sus errores y aciertos, sin las pretensiones de infalibilidad, de eficacia y eficiencia, propias de las otras modalidades de evaluación de las prácticas. (pp.11-12)

Por tanto, las prácticas pedagógicas han estado en permanente reconstrucción conceptual. En Colombia, son significativos los trabajos en torno a la práctica docente, como los adelantados por Baquero y Villa (2013), y que con anterioridad, han sido desarrollados por De Tezanos (1985) quien ha orientado sus estudios sobre la revisión de modelos de formación desde el análisis de dispositivos teóricos y prácticos en los currículos de las escuelas normales; Munévar, Quintero y Yepes (2000) quienes han hecho estudios de los dispositivos de formación desde la práctica docente como espacio de investigación; Quintero (2001) quien se ha interesado por la investigación acción en las prácticas; Sandoval y otros (2002) e Isaza, Henao y Gómez (2005) quienes se han interesado por desarrollar investigaciones situadas, orientadas a la reconstrucción histórica en contextos específicos de formación como las universidades. Estos autores, entre otros investigadores, se han ocupado de indagar sobre las prácticas y sus referentes didácticos o epistemológicos.

Son significativas, de igual manera, las investigaciones que se han preocupado por conceptualizar acerca de la práctica docente. Autores desde Doyle hasta Blanco (citados por Moreno, 2001), durante la década de los ochenta, han comprendido la práctica docente como una ocasión para aprender a enseñar, una experiencia planificada que hace uso de los conocimientos teóricos para ser aplicados en el proceso enseñanza-aprendizaje. Este es un proyecto integral 
que regula las relaciones de los alumnos con la escuela para poder relacionar la teoría y la práctica, hasta concebirlo como un componente de un programa de formación inicial en donde el estudiante va estableciendo sus esquemas de acción respecto a su función como profesor en los diferentes ámbitos que su quehacer le obliga.

En este mismo orden de ideas, los propósitos de la práctica que van en relación directa con la conceptualización que se tenga de la misma, han sido reconocidos por diversos investigadores durante la década de los ochenta desde Gimeno y Fernández (1980) hasta Blanco (1988) (citados en Moreno García, 2001, pp. 19-20), quienes destacan que la práctica es una experiencia para hacer significativos los conocimientos teóricos, la posibilidad de entender la escuela como una empresa colectiva, un ejercicio para aprender a resolver las rutinas ordinarias, ejercitar técnicas aprendidas, y un espacio para ir configurando estilos en el futuro profesor, adquirir aprendizajes relacionados con la cultura profesional y desarrollar la reflexión sobre la acción.

Wilfred Carr ratifica: "la imposibilidad de comprender el significado y la importancia de una práctica si no nos referimos a las intenciones de quien la realiza, pero también indica que dicho significado e importancia son una construcción social, histórica y política" (citado en López y Basto-Torrado, 2010 p. 280). Esto presupone un educador que no acepta de por sí las políticas y los modelos impuestos que se hacen válidos a través de dispositivos de control, sino que es un actor intelectual, reflexivo y crítico que confronta la realidad para dar respuesta a las nuevas exigencias pedagógicas y sociales que le propone la sociedad de la información y el conocimiento.

Los propósitos de la práctica docente de igual manera son relevantes dentro del proceso de formación inicial y por ello, el Ministerio de Educación Nacional en la Resolución 2041/16 (2016), sobre las "condiciones de calidad de los programas de licenciatura", define que dichos programas de formación deben diseñar sus contenidos curriculares y el desarrollo de competencias en el educador teniendo en cuenta los siguientes criterios:

asegurar que los estudiantes adquieran preparación en la práctica pedagógica. En ella, los estudiantes de Licenciatura [sic] deben comprender y apropiar las dinámicas del aula y su contexto, reconocer las diferencias y modalidades de formación de niños, niñas, adolescentes, jóvenes y adultos, y asociarla con la disciplina que se enseña y con las situaciones, eventos o fenómenos que dicha disciplina conlleva. [...] el educador debe tener formación específica en pedagogía, didáctica de los saberes escolares y las disciplinas, lo que le permitirá orientar procesos educativos, acompañar y promover la formación integral, los aprendizajes de los estudiantes, además del desarrollo de valores, tomando en consideración sus contextos particulares. [...] el currículo debe incluir igualmente, componentes formativos y espacios académicos 
dedicados a la práctica pedagógica y educativa, con la supervisión apropiada para apoyar su evaluación crítica en relación con los aprendizajes promovidos. [...] la institución de educación superior deberá organizar los programas de Licenciatura [sic] con el fin de formar en valores, conocimientos y competencias profesionales del educador, y en la utilización de buenas prácticas en la enseñanza de las disciplinas a su cargo, en los niveles educativos para los cuales se está formando. [...] los valores, conocimientos y competencias del educador comprenderán los siguientes cuatro componentes, que deben ser desarrollados conjuntamente, asegurando su articulación: 1. componente de fundamentos generales; 2. componente de saberes específicos y disciplinares; 3 . componente de pedagogía y ciencias de la educación, y 4. componente de didáctica de las disciplinas. (Resolución 02041, 2016)

Puede reconocerse que la formación inicial docente está centrada, en el sistema educativo colombiano hoy, en el desarrollo de competencias, en cuyo trasfondo está el paradigma conductista

que concibe la enseñanza como una actividad con base científica y a los profesores como profesionales competentes, ejecutores o aplicadores de los objetivos de entrenamiento, que se considera, garantizan un alto rendimiento. Por tanto, en su origen se encuentran teorías psicológicas conductistas, y en general, la epistemología positivista. (Pavié, 2012 p. 48)

En contracorriente a esta anterior mirada frente a la formación del docente, surge entonces el paradigma humanista, orientado a la indagación, que pretende instalarse como alternativo dentro de la fase inicial, porque en dicho paradigma subyace la idea de profesor, como sujeto reflexivo. Pero la realidad es que se continúa viendo la formación desde un modelo tecnológico, y esto, no permite afrontar la complejidad de los fenómenos educativos a los que se ve abocado el futuro profesor durante el desempeño de su práctica. Si se adopta un modelo que conlleve a la formación de un docente reflexivo, implica concebir las prácticas como eje central de desarrollo de la profesionalidad, sacándolas de espacios curriculares específicos, cerrados, preconcebidos, estáticos y considerarlas en toda su complejidad como el lugar por excelencia para aprender y construir conocimiento tanto de su saber específico como pedagógico, asumiendo la realidad escolar como un todo.

\section{Hallazgos}

El reconocimiento de las diversas miradas sobre la práctica docente en los anteriores contextos permite establecer cómo fue el desarrollo de la concepción de la práctica docente y cuál fue el horizonte de sentido que definió los procesos de formación que favorecen al educador en la apropiación de los fundamentos y los conocimientos para efectuar su labor profesional educativa. 
Entre las características de manera general que se pueden reconocer se encuentran las siguientes y que son de relevancia dentro del estudio:

i) La preocupación, a partir del surgimiento de la teoría crítica, por reconocer la práctica docente como campo de reflexión e investigación teniendo como referente la cotidianidad escolar, permitió al docente un lugar para poner en en escena de sus saberes. El salto cualitativo se da al pasar de un enfoque de práctica de transmisión-recepción a uno crítico y reflexivo.

ii) La práctica docente como campo de aplicación de conocimientos, métodos, disciplinas y técnicas permite un acercamiento entre los saberes adquiridos a partir de los contenidos teóricos propios de las asignaturas que hacen parte del currículo y su pertinencia en la práctica.

iii) La práctica docente como espacio académico favorece el desarrollo de la formación personal-profesional con un perfil amplio en donde el sujeto al involucrarse en los asuntos de la dinámica escolar construye conocimiento tanto de la escuela como de la docencia.

iv) La formación profesional docente está dada con base en el desarrollo de competencias.

\section{Consideraciones generales del programa de licenciatura en Filosofía en la Fundación Universitaria San Alfonso que fundamentan la propuesta de formación inicial docente}

Para la Fundación Universitaria San Alfonso, en su nueva mirada frente al saber disciplinar como profesional en la licenciatura en Filosofía, tal y como queda expresado en el documento maestro, el cual recibió aprobación de registro calificado por el Ministerio de Educación Nacional (2015, p. 112), destaca cómo el saber disciplinar filosófico en relación con su desarrollo práctico, implica reconocer que el ejercicio del conocimiento encierra un proceso de interacción discursiva entre quien lo posee, lo recrea y lo recontextualiza y aquel que lo apropia para la comprensión del mundo. Así, se convierte el docente en el protagonista de un discurso no hegemónico, cuyo desarrollo se propicia desde un enfoque hermenéutico-reflexivo, en donde los sentidos y significaciones de la práctica giran en torno a la resignificación del conocimiento y su aplicabilidad en contexto.

Entre los criterios generales que orientan el programa de Licenciatura en Filosofía de la Fundación Universitaria San Alfonso se encuentran los siguientes en orden de importancia. 


\section{Principios de la Fundación Universitaria San Alfonso}

\section{Educación integral cristiana}

La Fundación Universitaria San Alfonso entiende por formación integral aquella educación que pretende el desarrollo armónico de todas las dimensiones de la persona. Ella se dirige, entonces, a la formación de una persona que construye el saber en conjugación con el obrar moral; lo cual lleva al sujeto a la coherencia entre el saber, el ser y el hacer. Con esta premisa, que se hace explícita en el Proyecto educativo institucional (2016b), se posibilita la transformación de la sociedad según el ideal de vida y de cultura que, en sí misma, ha asumido como Institución con una gran responsabilidad social en los contextos donde tienen su actuar quienes allí se han formado como profesionales, en este caso, de la educación.

Para hacer posible la formación integral se requiere del auténtico conocimiento, que no es saber acumulado, sino visión de conjunto, anhelo de unidad-totalidad y, en cuanto tal, trasciende todas las ramas del conocimiento. Se destaca este principio en el Documento maestro de la licenciatura en Filosofía de la Fundación Universitaria San Alfonso (2016a) en donde se afirma que:

[e]sta formación integral pretende el logro de la autonomía personal, la adquisición y recreación del conocimiento, la aplicación del conocimiento a la solución de los problemas del país, la formación ética del profesional y la formación social, cívica y política de los ciudadanos. (p. 9)

Así, la formación integral se apropia en el programa de formación de licenciatura en Filosofía de la fundación como una formación general que busca que la persona sea capaz, amante de los desafíos del futuro, de tomar decisiones responsables en diversos ámbitos de su ejercicio profesional.

Esta dignidad ontológica se presupone con universalidad, válida para todos los hombres; hecho que trae como consecuencia un sentido de educación que se orienta al desarrollo del ser del hombre, de su capacidad como sujeto crítico, dialógico y transformador. Igualmente, esta dignidad ontológica se asume en la integralidad en tanto desarrollo del hombre en todas las dimensiones de la persona (material, intelectual, cultural, ética, de valores y de apertura al absoluto). Con esto, se propone una jerarquía de valores orientada a una educación desarrolladora que logre gradualmente los siguientes niveles:

\section{Educación de lo humano, desde lo humano y para lo humano}

Se espera formar un ser humano que vea en la afirmación de Dios la condición trascendente de la realización total del hombre y de sus valores. Este ser 
humano ha de encontrar el poder creciente y la vocación de transformación en el humanismo cristiano, y ha de concebir la ciencia y la tecnología como una muestra fecunda de la realidad cocreadora del hombre y de la voluntad misma salvífica de Dios y, por tanto, de apertura y respeto hacia la dignidad de cada persona en igualdad de condiciones.

\section{Orientación para ser más y no para tener más}

Se espera formar un ser humano que, sin caer en el desprecio del tener y de las condiciones materiales de la vida, valore el sentido existencial que tiene "lo humano" y se abra a un concepto de ética acorde al dinamismo de la cultura; de la vida intelectual, educativa, filosófica y religiosa. Con ello, el programa de licenciatura en Filosofía se ocupa del sentido de lo ético en términos de la producción y manejo del conocimiento.

\section{Formación de una actitud crítica}

Se espera formar un ser humano que esté en capacidad de cuestionar todas las situaciones y estructuras que alienan y deshumanizan, en especial las que provienen del abuso del poder, de la explotación de los trabajadores y de la injusticia provenientes de las relaciones con otros países más desarrollados. Este panorama deseable se forja con el desarrollo de la capacidad de discernimiento y toma de decisiones frente a las alternativas que se puedan brindar a una situación, a un problema, a un hecho profesional. Así, el programa de licenciatura en Filosofía reconoce en el discernimiento la potenciación de la actitud crítica de los profesionales que ejercen su quehacer en el marco del libre-pensamiento sustentado con criterios debidamente argumentados.

\section{Pedagogía para la convivencia social y el respeto a los demás}

Se espera formar un ser humano que cultive la idea de la alteridad, del bien común, de la paz, de la justicia y de la solidaridad. Por ello, el programa de licenciatura en Filosofía asume la práctica docente como una instancia fundamental para mostrar la coherencia entre el ser, el sentir, el hacer y el convivir; donde se hace reconocimiento del sujeto, esa Otredad, desde la ética del cuidado.

\section{Capacitación para superar la pobreza}

Se espera formar un ser humano que logre satisfacer las necesidades fisiológicas, psicológicas y espirituales, orientando desde un comienzo la formación hacia la superación del egoísmo y al reconocimiento del valor y los derechos del otro. El programa de licenciatura en Filosofía cimienta en sus profesionales la responsabilidad con el cuidado de sí y del otro en términos de intercambios 
equitativos, tolerantes, solidarios, respetuosos en los diferentes contextos de desarrollo de su proyecto de vida.

\section{Educación para la vida}

La Fundación Universitaria San Alfonso, como proyecto educativo, busca facilitar el acceso de sus estudiantes a la cultura sistemática en los campos de la educación, la filosofía, la teología, la psicología, la comunicación social, el trabajo social, la utilización de las tecnologías para que, al egresar, estén en condiciones de participar activamente en el mejoramiento de los niveles de vida y en el cambio social fundamentado en el mensaje cristiano.

Así, la Fundación Universitaria San Alfonso estructura su proyecto educativo (PEI) como resultante de un proyecto de cultura para la formación, con miras a contribuir a la construcción de una nueva sociedad constituida por seres humanos que actúen con un sentido del ejercicio responsable de la libertad, de la equidad, de la participación en los procesos sociales, de la justicia social y de la tolerancia en las ideas.

El proyecto educativo de la Fundación Universitaria San Alfonso analiza con profundidad y con los métodos propios de cada una de las disciplinas académicas, la realidad actual del mundo y de la sociedad contribuyendo de esta manera al enriquecimiento del saber humano. La orientación que se da en los diferentes programas académicos, por otra parte, hace que la educación no sea puramente de corte academicista. La capacitación pedagógica y formativa implica una visión determinada de la persona, de la sociedad y de la cultura. Todo ello pensando desde el horizonte de los temas, los problemas y los desafíos de la cultura latinoamericana y colombiana

\section{Principios fundamentales, que soportan el proyecto educativo institucional}

\section{La dignidad de la persona}

Se concibe la persona humana como un ser integral y armónico, hijo de Dios y digno de respeto. Por ello, la Fundación Universitaria San Alfonso, se compromete con la persona por medio del servicio, el testimonio y la solidaridad; con la promoción de una cultura del amor y la fraternidad y del rechazo de todo tipo de violencia e injusticia social. A la base de todo este proyecto, como institución católica, está el Evangelio, el Pensamiento Social de la Iglesia y la espiritualidad de San Alfonso María de Ligorio, que inspira la acción pastoral como carisma redentorista. 
Por eso, el mensaje de Cristo es el criterio fundamental que orienta esta Institución de Educación Superior (IES) en sus programas, en la investigación y en el servicio a la sociedad. Así, la Fundación Universitaria San Alfonso (2016a) siempre busca que:

los alumnos se formen para una mayor libertad, solidaridad y responsabilidad social y adquieran una visión ética del mundo que los comprometa con el respeto permanente de los derechos humanos, la observancia de sus deberes personales, familiares y sociales, la participación ciudadana y la promoción constante de ésta, la realización de la justicia en todas sus dimensiones y áreas, y el mejoramiento efectivo de la calidad de vida de las personas en particular, y de las comunidades en general. (p. 12)

\section{La promoción humana}

La Fundación Universitaria San Alfonso (2016a) tiene como preocupación fundamental que las personas y las comunidades participantes de este proyecto, logren una formación integral que les permita alcanzar mejores condiciones humanas de vida. Por eso:

se promoverá el comportamiento ético, la equidad y la igualdad de oportunidades, el respeto por el otro y el reconocimiento de la diferencia, el manejo del conflicto y la tolerancia, el trabajo en equipo y la cultura de diálogo, la interdisciplinariedad, la solidaridad y la justicia social. (p. 12)

Al mismo tiempo, la Fundación Universitaria San Alfonso en su Proyecto educativo institucional (2016b) capacita a sus alumnos en:

el estudio de los graves problemas y conflictos contemporáneos tales como, la dignidad de la vida humana, la promoción de la justicia para todos, la calidad de vida personal y familiar, la protección de la naturaleza, la búsqueda de la paz y de la estabilidad política, una distribución más equitativa de los recursos del mundo y un nuevo ordenamiento económico y político que sirva mejor a la comunidad humana a nivel nacional e internacional. (p.1)

\section{El desarrollo humano y social}

Conscientes que la promoción integral es posible con educación para todos, la Fundación Universitaria San Alfonso propende por la educación que conduce al desarrollo humano y social de sus educandos. En efecto, en su marco filosófico - expuesto en el proyecto educativo-, la Fundación Universitaria San Alfonso garantiza la excelencia, la calidad y la interdisciplinariedad como condiciones para lograr la capacidad de asumir con responsabilidad los desafíos de la sociedad, la creatividad para iniciar procesos nuevos de transformación y la responsabilidad frente a las tareas que va asumiendo en las comunidades y en la sociedad. 


\section{La flexibilidad conceptual, metodológica y administrativa}

La Fundación Universitaria San Alfonso (2016a) comprende perfectamente:

la necesidad de vivir y promover la apertura de criterios, métodos y procesos de gestión, para un auténtico desarrollo institucional y el logro de la misión fundacional. Se parte de la creencia de que sólo [sic] así se está promoviendo la creatividad, la innovación, la producción de conocimiento, y, en últimas, el desarrollo integral de las personas y comunidades a las que servirá. (p.13)

\section{La promoción de los valores culturales nacionales y latinoamericanos}

De igual manera la Fundación Universitaria San Alfonso (2016a), como lo sigue exponiendo en su documento maestro, señala que se promueve en los estudiantes una actitud de:

[u]n profundo amor a su territorio y un deseo de ampliar sus horizontes de progreso, en la búsqueda de un país justo, sin violencia, en pleno desarrollo y progreso para todos, en la búsqueda de la construcción de una "ciudad ideal"; la Fundación Universitaria San Alfonso, promueve los valores culturales que configuran la identidad de Colombia y de América Latina abierta al mundo entero, ratificando con ello una voluntad férrea de servicio a la sociedad. (p. 13)

\section{Enseñar aprendiendo}

Continuando con el análisis sobre los principios que orientan el Proyecto educativo institucional y que los esboza en el Documento maestro de la licenciatura en Filosofía de la Fundación Universitaria San Alfonso (2016a), como se viene mencionando, se reconoce entonces que: "la docencia es un proceso dinámico y, como tal, requiere del análisis perseverante de los distintos factores que intervienen en el mismo y que desde luego son susceptibles de mejorar e innovar" (p.13).

Atendiendo a esta concepción de igual manera para la Fundación Universitaria San Alfonso:

El proceso docente supone un vínculo inter e intrahumano preciso cuyo tejido profundo lo conforma la cultura, es decir, la combinación de convicciones, disposiciones y conductas vinculados con elementos de conocimiento científico y con principios que tienen que ver con saber hacer, saber juzgar, saber vivir y poder apreciar. (p. 14)

Esta trama del saber y la cultura es la materia de la que brotan las destrezas en el orden del conocer, decidir, valorar y transformar que se consideran insustituibles para guiar la educación. En esta óptica, para la Fundación Universitaria San Alfonso en su Proyecto educativo institucional (2016a) la labor docente se propone:

[e]nseñar las ciencias conforme a criterios de verdad y criticidad. [...] Alcanzar competencia en el manejo de distintas situaciones y experiencias. [..] Desarrollar la 
capacidad práctico - moral según criterios de responsabilidad y justicia.

[...] Consolidar destrezas técnicas para obrar con precisión y eficiencia. [...] Cultivar

la sensibilidad y creatividad de acuerdo con pautas estéticas. (pp. 34-35)

Se reafirman cada uno de estos principios plasmados en el Documento maestro de la licenciatura en Filosofía de la Fundación Universitaria San Alfonso (2016a) cuando se reconoce que:

[l]a actividad de estudiantes y docentes está enmarcada en los currículos. Estos organizan e integran oportunidades, experiencias y actividades de enseñanza y de aprendizaje, según áreas técnicas, líneas de investigación que se refieran a los propósitos definidos de formación en una disciplina o profesión. El diseño, el desarrollo y la realización de los currículos deben favorecer la formación integral del estudiante. (p. 14)

\section{La práctica investigativa como condición emergente para la formación docente}

La discusión que surge de revisar los modelos que prevalecieron a lo largo de la historia de las prácticas docentes en nuestro contexto y lo que la IES propone desde sus referentes pedagógicos, epistemológicos y misionales condensados en su proyecto educativo, afianza la investigación como el "corazón" del trabajo universitario, manteniendo fuertes vínculos con la docencia y el servicio a la sociedad. Para la Fundación Universitaria San Alfonso la búsqueda del saber amplía las fronteras del conocimiento y de su aplicación. Así, el estudiante cambia sus pautas de conducta, transforma sus esquemas por medio del aprendizaje; él mismo investiga, analiza y critica el conocimiento. Esta tarea de reflexión constante y autónoma de la búsqueda de respuestas o propuestas conduce a la enseñanza de calidad.

La investigación y la docencia requieren de la creación y la recreación del conocimiento; de la primera, para generar nuevos conocimientos y, de la segunda, para su difusión, su asimilación y la innovación por parte del estudiante.

Las investigaciones de corte interdisciplinario son una exigencia hoy. Estas se convierten en una especial tarea para la generación de nuevo conocimiento en las áreas del saber propias de las disciplinas donde se están formando inicialmente los futuros profesionales de la educación. Además, en procura de dar respuesta a las condiciones de calidad que exige la norma, un docente crítico-reflexivo debe asumir su práctica docente como práctica investigativa que procure dar respuesta a los problemas presentes en el contexto; su desarrollo científico e intelectual estará precedido por la problematización de ciertos asuntos que encierran el objeto propio de la pedagogía y la didáctica: la educación, el proceso enseñanza-aprendizaje y el proceso docente educativo. 
Por ello, la Fundación Universitaria San Alfonso busca que los proyectos de investigación conduzcan a la integración de los saberes disciplinares y laborales que puedan ofrecer respuesta a las situaciones que están experimentando las comunidades donde se lleva a cabo la actividad docente para que se conviertan en comunidades de sentido.

\section{Los criterios pedagógicos}

La Ley 115 (1994) presenta "la educación como un proceso de formación permanente, personal, cultural y social, que se fundamenta en una concepción integral de la persona humana, de su dignidad, de sus derechos y deberes" (art. 1).

El fundamento de la educación hay que colocarlo en el hecho evidente de que tanto el ser humano como la sociedad, la cultura y la historia, están en proceso de realización y perfeccionamiento. Estas son posibilidades abiertas al futuro deseable, y su destino está en manos de la práctica educativa y, por supuesto, de la pedagogía que es la manera propia de desarrollar y aplicar la educación.

Los modelos y los criterios pedagógicos elegidos establecen el tipo de educación que se está ofreciendo. Estos modelos y criterios señalan el tipo de persona, de sociedad deseada, los valores que deben ser implementados, la selección de conocimientos que deben ser enseñados, las metodologías que deben ser aplicadas y el horizonte de la utopía histórica de los pueblos.

La institución asume los siguientes criterios pedagógicos:

i) El acto pedagógico es entendido como una interrelación entre personas autónomas y libres, y debe ser realizado en el contexto de las relaciones sociales y estar fundamentado en el respeto mutuo.

ii) Cada persona es responsable de su autorrealización y maduración; por tanto, el acto educativo no sustituye al sujeto, ni condiciona su libertad ni excluye su responsabilidad.

iii) El acto educativo en el proceso de enseñanza-aprendizaje, impulsa, orienta y favorece el proceso de autorrealización personal y profesional.

iv) La formación ofrecida debe tener en cuenta la integralidad de la persona, la relación personal y social, la dimensión histórica y el sentido de trascendencia.

v) El desarrollo de aquellas habilidades, capacidades, actitudes y aptitudes conducentes a un desempeño profesional excelente.

vi) El respeto por la autonomía del saber y la legitimidad de la ciencia. 
vii) La implementación de la práctica pedagógica de la investigación formativa en la diversidad de modalidades.

viii) La formación del espíritu investigativo que impulsa el deseo del saber autónomo, reflexivo y crítico.

ix) El fortalecimiento de la interdisciplinariedad que ofrece una visión compleja y diversificada de los temas y problemas de la realidad, así como la amplitud de propuestas para buscar soluciones.

x) El desarrollo de aquellas competencias cognitivas, comunicativas, sociales e investigativas, que son propias de cada profesión.

xi) La elaboración y aplicación de currículos contextualizados y de planes de estudio pertinentes, que respondan a necesidades, problemas y soluciones acordes con la realidad social, histórica y cultural del país y del contexto internacional.

xii) La apertura al sentido global.

xiii) El fomento del diálogo entre la razón y la fe.

xiv)La valoración del conocimiento y de la verdad, elaborados desde la perspectiva de la fe.

xv) La institución es de confesionalidad católica, sin embargo profesa un profundo respeto por la libertad de conciencia.

xvi)La implementación de la cultura de la autoevaluación y autorregulación.

xvii) La aplicación de metodologías activas que favorezcan el trabajo personalizado, la investigación, la participación y la comunicación del conocimiento.

xviii) El fomento de habilidades y destrezas para la utilización de los recursos bibliográficos y tecnológicos de punta.

xix)La formación de los docentes para responder a las exigencias académicas.

El programa académico profesional de licenciatura en Filosofía analiza críticamente una compleja trama de problemas filosóficos y pedagógicos conectados articuladamente en las distintas asignaturas del mismo programa, lo cual, permite implementar una formación integral profesional coherente con la complejidad epistemológica y ontológica de la cultura reinante en la actualidad, respondiendo así a las exigencias de la formación superior de un licenciado. 
De esta manera, el nivel de complejidad del programa propuesto corresponde con la formación profesional en educación superior. Es así, que la licenciatura en Filosofía le proporciona al egresado los elementos fundamentales para poder interactuar armónica y dialógicamente con otros tipos de saberes, fortaleciendo el aspecto investigativo en el campo pedagógico, didáctico y metodológico, logrando de este modo, un intercambio interdisciplinar ajustado a las demandas en el nivel académico y ocupacional, para promocionar el saber filosófico según los parámetros o requerimientos de los proyectos históricos de la sociedad colombiana, en un contexto globalizado.

\section{Concepción de práctica docente en la licenciatura de Filosofía en la Fundación Universitaria San Alíonso}

\section{Definición operativa de la práctica docente en perspectiva dialéctica}

La Fundación Universitaria San Alfonso, en su nueva mirada frente al saber disciplinar como al pedagógico en la licenciatura en Filosofía, destaca como el saber disciplinar filosófico en relación con su desarrollo práctico implica reconocer que el ejercicio del conocimiento encierra un proceso de interacción discursiva entre quien lo posee, lo recrea y lo recontextualiza, y aquel que lo apropia para la comprensión del mundo.

Así, se convierte el docente en el protagonista de un discurso no hegemónico, cuyo desarrollo se propicia desde un enfoque hermenéutico-reflexivo, en donde los sentidos y las significaciones de la práctica giran en torno a la resignificación del conocimiento y su aplicabilidad en contexto. Se reconoce entonces la práctica docente como un espacio de encuentro del docente en formación con el fenómeno educativo a través de diferentes teorías, enfoques y orientaciones que requieren el acceso a procesos de reflexividad, contextualización y apropiación tanto del saber cómo de la forma de facilitar la retención y aplicación de este.

Reconociendo los diferentes criterios sobre los cuales se define el sentido y la significación de la práctica docente, se hace necesario estructurar un modelo de práctica docente que ofrezca una respuesta real a los planteamientos que se encuentran especificados en los diferentes documentos institucionales y que sirva de orientación tanto para el diseño curricular de las áreas involucradas en el componente pedagógico, como para la puesta en marcha de una práctica social e históricamente contextualizada.

En lo que respecta a la formación profesional y en virtud de las funciones sustantivas propias de la institución de educación superior: docencia, investigación y proyección social, resulta de gran interés vincular en el área disciplinar los componentes pedagógico e investigativo, y dentro del primer componente la asignatura de práctica docente como un concepto base y orientador del diseño 
curricular de dicha área con el propósito de ubicar relaciones de integración entre el saber disciplinar y su orientación hacia la formación profesional del futuro docente en perspectiva investigativa. Desde esta mirada investigativa, la institución formadora acompaña el desarrollo o la participación del estudiante en formación inicial en los proyectos educativos en contexto, potenciando de esta manera su interés por el diseño de currículos. Pone al servicio de esa comunidad de sentido su experiencia de aprendizaje.

En esta trabajo de investigación interesa estudiar el proceso de práctica docente en la formación de los licenciados en filosofía de la Fundación Universitaria San Alfonso de Bogotá, Colombia, lo cual supone profundizar en el análisis de la misma, determinando fortalezas, intencionalidad pedagógica, valoración e impacto, lo que a su vez ayuda en la definición de las diferentes posibilidades de transformación de las demandas sociales actuales como de las exigencias normativas y, estas últimas en relación con las condiciones de calidad.

El programa ofrece a sus estudiantes el título de licenciado en filosofía, el cual corresponde con la formación impartida de modo pertinente según cuatro ejes problémicos presentados en forma de binomios (naturaleza y lenguaje, conocimiento y aprendizaje, crítica y realidad, ética y acción humana), desglosados a su vez en cuatro áreas fundamentales (humanística, disciplinar, lenguajes, y profundización), las cuales desarrollan los distintos componentes como son (histórico, sistemático, socio-político, pedagógico, investigativo, escrituras, lengua extranjera e informática), integrados en dos ciclos de formación (básica y profesional), conformados a su vez por diferentes asignaturas.

De esta manera el programa de licenciatura en Filosofía, orientado por el carisma alfonsiano persigue una formación integral basada en su condición humana, disciplinar pedagógica, política, cultural, social, ética, estética, laboral, sustentada en el quehacer del educador desde un horizonte filosófico constituido por los criterios teóricos y metodológicos del humanismo. Sus contenidos se orientan por el aprendizaje autónomo, flexible y colaborativo de las áreas de formación disciplinar, profesional e investigativa y, de los ciclos de formación básica y de profundización a través de los ejes problémicos anteriormente mencionados.

Por su parte, Bustamante (2006) menciona que:

[1]a formación docente no puede ser una mera revisión de fórmulas didácticas o un adiestramiento en disciplinas específicas, tiene que ser el espacio que acoja la inquietud del profesor por trascender, el lugar en donde, mediante la reflexión, pueda aclarar su posición, respecto de la problemática educativa, su rol en la dinámica social, su forma de entender el mundo" (citado en Ministerio de Educación, 2013, p. 49) 
Lo anterior permite la comprensión de la práctica docente desde una mirada hermenéutica y reflexiva, que presupone la reconstrucción de la misma y del propio pensamiento, a través del proceso de indagación e interpelación continua de la realidad tanto a nivel interno como externo al aula de clase.

Se convierte entonces la práctica docente en un espacio donde es posible la deliberación y el consenso con miras a la construcción colectiva de los diferentes saberes que permean el escenario educativo.

En conclusión, el objetivo central de la práctica docente para el programa de licenciatura en filosofía de la Fundación Universitaria San Alfonso es promover el desarrollo profesional del practicante a partir del análisis y la reflexión sobre la práctica pedagógica que le permita reconocerse como intelectual de la educación y agente de cambio sociocultural, asunto que se ve reflejado en el perfil profesional del licenciado en filosofía quien a través de su proceso formativo educativo, se preocupa por confrontar la realidad e incursionar en todos los terrenos de la vida tanto intelectual como social y política, desde una perspectiva humanista y de rigurosa reflexión comprometiéndose así con la construcción y transformación social.

Tomando en consideración los criterios que se han abordado con anterioridad sobre la práctica docente y habiendo hallado aquellos elementos distintivos que se implican en el concepto y, consecuentemente, en su expresión en la práctica dentro de los programas de formación del futuro profesional en educación, se define operativamente en esta investigación la práctica docente desde una perspectiva dialéctica como: el espacio pedagógico conscientemente concebido, organizado de manera secuencial (práctica inicial, práctica guiada y práctica autónoma) y controlado, que se ubica curricularmente en la formación inicial del profesional de la educación, cuyo objetivo directo es propiciar el escenario donde el debe integrar en la práctica aquellos conocimientos que en el orden teórico ha adquirido y, donde debe evidenciar en su accionar la apropiación de los mismos tanto en las disciplinas referidas a la ciencia que estudia, como aquellas de orden didáctico e investigativo.

Encuentra de esta manera sentido y significación el docente en formación inicial a todos los procesos académicos desarrollados durante la licenciatura, al sentirse en capacidad de expresarlos en su accionar en variados contextos, con sus diversas finalidades de aprendizaje, los cuales se caracterizan por la interculturalidad y convocan a la reflexión situada sobre las manifestaciones propias de la vida cotidiana escolar, asumiendo el compromiso como agente educativo responsable de fomentar el cambio y la transformación en las instituciones educativas donde realiza el ejercicio de la práctica como parte esta de las mismas contradicciones que en el ámbito se hacen presentes. 


\section{Conclusiones}

A partir del análisis de los diferentes aspectos que encierra el desarrollo profesional docente y específicamente en lo que tiene que ver en torno a la relación entre el saber disciplinar y la profesionalización se puede reconocer que, el desarrollo profesoral ha recorrido un largo camino, que va desde el acceso a procesos enmarcados en principios de la ciencia positiva con tendencias conductistas y moralistas, pasando por la práctica docente institucionalizada, hasta la consolidación de una cultura de la profesión, marcada mucho más por la estructuración de la pedagogía como campo científico, al definir pautas de orden epistemológico, metodológico, práxico e investigativo. Asuntos significativos en el campo de formación de los educadores hoy, y que se establecen como principios conducentes a perfilar un profesional que reconozca además de sus roles tradicionales, su condición como mediador cultural, reflexivo, crítico, negociador de significaciones e intelectual, para poder confrontar los retos pedagógicos y sociales que le demanda el medio.

En el mismo sentido, las prácticas docentes han ido ganando espacio en la formación inicial desde criterios que, en un primer momento, se definen desde un concepción tradicional del oficio a través del esquema: información-observación- imitación de profesores experimentados, pasando por la concepción personalista cuando se reconocen las prácticas como el espacio para el desarrollo integral —en perspectiva humanista - del futuro profesor y por último la concepción orientada a la indagación: profesor investigador, práctica investigativa.

Otro aspecto por señalar es la concepción que se tiene frente a la práctica docente cuya definición se ha visto condicionada por movimientos sociopolíticos, económicos y culturales que las sociedades han experimentado en un tiempo específico, y cuyo horizonte de sentido sirve de marco para el diseño de política pública específicamente en el campo de la educación y dentro de este en el sistema de formación de los educadores.

Atendiendo a esta dinámica se puede establecer desde una evolución histórico pedagógica lo siguiente: la existencia de tres grandes tendencias curriculares dominantes que orientan el ejercicio de la práctica en el proceso formativo: técnica, práctica y emancipatoria atendiendo al tipo de interés que mueven las acciones humanas en la definición de procesos identitarios en los que subyace unas formas de organización, diseño y desarrollo de la practica como experiencia docente.

Otro aspecto es el desempeño de la labor docente en un inicio enmarcada en procesos de dependencia a corrientes epistemológicas, didácticas, metodológicas, de planificación, hasta lograr actualmente procesos de autonomía que 
conllevan a su asunción como profesional e intelectual de la educación y, por tanto, a la construcción social de la profesión.

En consonancia, el estudio sobre las prácticas docentes como dispositivo de formación se asume desde diferentes perspectivas: una netamente técnica; otra que hace hincapié en procesos reflexivos y otra en comprender la práctica como experiencia y acontecimiento según Baquero y Villa (2013), desde una "formación en las causas" como lo menciona Aracely de Tezanos (1986).

Por otra parte, atendiendo a lo que la IES pretende en específico, pero que puede entrar en diálogo con otras facultades de educación del país, es la construcción de su propio modelo pedagógico para la práctica docente y, en consonancia con lo expresado por su modelo educativo frente a: la formación profesional docente; la compresión significativa de la formación integral; la formación desde los principios del humanismo cristiano; concebir al profesional como sujeto histórico y transformador de su entorno desde una perspectiva crítica y por tanto propositiva.

Lo anterior significa que el docente en formación inicial, al concebir su práctica históricamente situada, puede hacer una mirada crítica e integral al sistema educativo, ya que él es en sí mismo producto del sistema, y este último se retroalimenta de lo que le aporta. De ahí que, se ubique desde el horizonte institucional una mirada muy especial hacia el mejoramiento de la formación de los docentes, lo que implica tener en cuenta los requerimientos de la sociedad, los avances de la ciencia y la tecnología en el campo de la innovación y el desarrollo.

Preparar entonces al futuro docente en su proceso de formación inicial implica, por una parte, atender a los presupuestos epistemológicos en los cuáles se enmarcan los lineamientos de política en el sistema educativo como son los esgrimidos en el discurso de las competencias; y, por otra, tener en cuenta el carácter misional de la institución, su cadena de valor, su clara orientación pedagógica y epistemológica.

Estas dos anteriores situaciones se circunscriben en una clara convergencia entre lo individual y social, en aspectos relacionados con lo cognitivo, socioafectivo y práxico (pedagogía dialogante), cuyo resultado será el de lograr una capacidad de comprensión y transformación del entorno que le ha sido asignado como espacio de aprendizaje como es el de la práctica docente.

Tomando conciencia de esta orientación que experimenta a lo largo de su programa de licenciatura podrá estimular la creatividad y la innovación para poder dar respuesta a los retos que el entorno reclama desde una perspectiva compleja, inter y transdisciplinar capaz de poder superar los modelos técnicos, artesanales, de administración curricular e incursionar en los que pretenden dejar de lado la 
transmisión del conocimiento y pasar a la transformación del entorno, a través de, los principios otorgados por el humanismo que permiten responder a la dinámica cambiante y contradictoria de la realidad.

\section{Referencias}

Anacona, M., Fayad, J., Granada, I., Gutiérrez, O., Medina, M., Núñez M., Posso, C., Rodríguez, S., Delgado, L., Jaramillo, N., Larroche, C. y Vallejo, C. (2007). Políticas públicas, saber pedagógico y prácticas pedagógicas en la Escuela Normal Superior Farallones de Cali. 1975-2005. En M. Baracaldo (coord.), Investigación de los Saberes Pedagógicos (pp. 55-117). Ministerio de Educación Nacional. https://es.slideshare.net/viteriange/investigacion-de-los-saberes-pedaggicos-men

Angulo, M. y Álvarez J. (2010). Tres dimensiones de la práctica pedagógica en la Facultad de Ciencias de la Educación de la Universidad de la Salle. Revista de la Universidad de la Salle, (51), 101-116. https://ciencia.lasalle.edu.co/ruls/vol2010/iss51/5/

Baquero, P. (2007). Práctica Pedagógica. Investigación y formación de educadores. Revista de Investigación, 7(1), 9-18. https://www.redalyc.org/pdf/952/95270102.pdf

Baquero, P. y Villa, W. (2013). Un visitante en el aula. Representaciones de la práctica pedagógica de formación docente. Editorial Universidad Distrital Francisco José de Caldas.

Bedoya, I. (2008). Pedagogía ¿Enseñar a pensar? Ecoe Ediciones.

Davini, C. (2008). Acerca de las prácticas docentes y su formación. Ministerio de Educación de Argentina.

De Tezanos, A. (1986). Maestros Artesanos Intelectuales. Estudio crítico sobre su formación. Universidad Pedagógica Nacional. Centro de Investigaciones.

Fundación Universitaria San Alfonso (2016a). Documento maestro licenciatura en Filosofía.

Fundación Universitaria San Alfonso (2016b). Proyecto educativo institucional. Editorial Kimpres.

Ministerio de Educación Nacional (2013). Sistema Colombiano de formación de educadores y lineamientos de política. Ediciones MinEducación.

Ministerio de Educación Nacional (2016, 3 de febrero). Resolución 02041 de 2016. Diario Oficial n. 49776. https://www.mineducacion.gov.co/1759/articles-356144_recurso_1.pdf

López, B. y Basto-Torrado, S. (2010). Desde las teorías implícitas a la docencia como práctica reflexiva. Educación y Educadores, 13(2), 275-291. https://educacionyeducadores.unisabana.edu.co/index. php/eye/article/view/1699

Moreno P. (2001). Lineamientos teóricos de la Práctica Educativa para los proyectos curriculares de la Universidad Pedagógica Nacional. Universidad Pedagógica Nacional. http://institucional.pedagogica.edu.co/admin/UserFiles/documento_pedagogico_09.pdf

Pavié, A. (2012). Las competencias profesionales del profesorado de lengua castellana y comunicaciones en Chile: aportaciones a la formación inicial [tesis doctoral, Universidad de Valladolid]. Repositorio Institucional, Universidad de Valladolid. http://uvadoc.uva.es/handle/10324/2794

República de Colombia (1994, 8 de febrero). Ley 115. Por la cual se expide la ley general de educación. Diario Oficial n. ${ }^{\circ}$ 41214. https://www.mineducacion.gov.co/1621/articles-85906_archivo_pdf.pdf

Schön, D. (1982). El profesional reflexivo. Cómo piensan los profesionales cuando actúan. Paidós. 